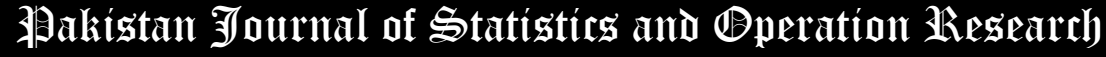

\section{Some Results on Exponentiated Weibull Distribution via Dual Generalized Order Statistics}

\author{
M. Alam ${ }^{1}$, R.U. Khan ${ }^{2}$, M.A. Khan ${ }^{3 *}$
}

* Corresponding Author

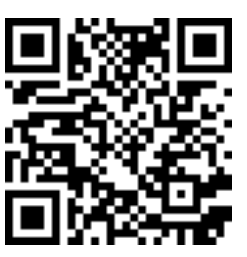

1. M. Alam, Department of Statistics and Operations Research, Aligarh Muslim University, Aligarh-202 002, India, mahfooz.stats@gmail.com

2. R. U. Khan, Department of Statistics and Operations Research, Aligarh Muslim University, Aligarh-202 002, India, aruke@ rediffmail.com

3. M.A. Khan, Department of Statistics and Operations Research, Aligarh Muslim University, Aligarh-202 002, India, khanazam2808@gmail.com

\begin{abstract}
In this paper, we use the concept of dual generalized order statistics dgos which was given by Pawlas and Syznal (2001). By using this, we obtain the various theorems and some relations through ratio and inverse moment by using exponentiated-Weibull distribution. Cases for order statistics and lower record values are also considered. Further, we characterize the exponentiated-Weibull distribution through three different methods by using the results obtained in this paper.
\end{abstract}

Key Words: Dgos, exponentiated Weibull distribution, order statistics, lower record values, ratio and inverse moments, truncated moment.

Mathematical Subject Classification: 62G30, 62E10, 60E05.

\section{Introduction}

The exponentiated Weibull distribution was proposed by Mudholkar and Hutson (1996). For more distributional properties of the exponentiated Weibull distribution, we may refer to Mudholkar, Srivastava and Freimer (1995) and Nassar and Eissa (2003).

A random variable $X$ is said to have exponentiated Weibull distribution (Mudholkar and Hutson (1996)) if its probability density function $(p d f)$ is of the form

$$
f(x)=\alpha \theta x^{\alpha-1}\left(1-e^{-x^{\alpha}}\right)^{\theta-1} e^{-x^{\alpha}}, \quad x \geq 0, \quad \alpha, \theta>0
$$

with distribution function $(d f)$

$$
F(x)=\left(1-e^{-x^{\alpha}}\right)^{\theta}, \quad x \geq 0, \quad \alpha, \theta>0 .
$$

The exponentiated Weibull distribution is the extended form of the two parameters weibull distribution. Also, it shows many characteristics quite similar to exponential; Weibull and exponentiated exponential distributions and their $d f$ and the $p d f$ are found to have closed forms. We can apply this distribution even on censored data.

Pawlas and Syznal (2001) introduced the concept of lower generalized order statistics lgos which enables have an idea about reverse order statistics and lower record values. Later, Burkschat etal. (2003) extensively studied this concept.

Let $X^{*}(r, n, m, k), r=1,2, \ldots, n$, be the $r$-th $d g o s$ and their joint $p d f$ is of the from 


$$
k\left(\prod_{j=1}^{n-1} \gamma_{j}\right)\left(\prod_{i=1}^{n-1}\left[F\left(x_{i}\right)\right]^{m_{i}} f\left(x_{i}\right)\right)\left[F\left(x_{n}\right)\right]^{k-1} f\left(x_{n}\right)
$$

for $F^{-1}(1)>x_{1} \geq x_{2} \geq \ldots \geq x_{n}>F^{-1}(0)$.

For the case $m_{i}=m, i=1,2, \ldots, n-1$, the $p d f$ of $r-$ th $d g o s X^{*}(r, n, m, k)$ is given

$$
f_{X^{*}(r, n, m, k)}(x)=\frac{C_{r-1}}{(r-1) !}[F(x)]^{\gamma_{r}-1} f(x) g_{m}^{r-1}(F(x))
$$

and the joint $p d f$ of $X^{*}(r, n, m, k)$ and $X^{*}(s, n, m, k)$, is

$$
\begin{aligned}
f_{X^{*}(r, n, m, k), X^{*}(s, n, m, k)}(x, y)= & \frac{C_{s-1}}{(r-1) !(s-r-1) !}[F(x)]^{m} f(x) g_{m}^{r-1}(F(x)) \\
& \times\left[h_{m}(F(y))-h_{m}(F(x))\right]^{s-r-1} f(y)[F(y)]^{\gamma_{s}-1}, \quad x>y,
\end{aligned}
$$

where

$$
\begin{gathered}
C_{r-1}=\prod_{i=1}^{r} \gamma_{i} \\
h_{m}(x)= \begin{cases}-\frac{1}{m+1} x^{m+1}, & m \neq-1 \\
-\log x, & m=-1\end{cases}
\end{gathered}
$$

and

$$
g_{m}(x)=h_{m}(x)-h_{m}(1), \quad x \in(0,1) .
$$

Several authors utilized the concept of $d g o s$ in their work. References may be made to Pawlas and Szynal (2001), Ahsanullah (2004, 2005), Mbah and Ahsanullah (2007), Khan et al. (2006, 2010), Khan and Kumar (2010, 2011) and Khan and Khan (2015) among others. In this paper, we mainly focus on the study of dgos arising from the exponentiated Weibull distribution.

\section{Some Theorems and Useful Results}

Note that for exponentiated Weibull distribution $f(x)$ and $F(x)$ satisfy the relation

$$
\alpha \theta F(x)=x^{1-\alpha}\left(e^{x^{\alpha}}-1\right) f(x) .
$$

The relation in (6) will be used to derive some simple recurrence relations for the moments of $d g o s$ from the exponentiated Weibull distribution.

We shall first establish the exact expression for $E\left[X^{* j}(r, n, m, k)\right]$. Using (4), we have,

\section{Relation for Inverse Moments}

$$
\begin{array}{r}
E\left[X^{* j-\alpha}(r, n, m, k)\right]=\frac{C_{r-1}}{(r-1) !} \int_{0}^{\infty} x^{j-\alpha}[F(x)]^{\gamma_{r}-1} f(x) g_{m}^{r-1}(F(x)) d x \\
=\frac{C_{r-1}}{(r-1) !} \Phi_{j-\alpha}\left(\gamma_{r}-1, r-1\right),
\end{array}
$$

where

$$
\Phi_{j}(a, b)=\int_{0}^{\infty} x^{j}[F(x)]^{a} f(x) g_{m}^{b}(F(x)) d x .
$$

Case I: when $m \neq-1$ 
On expanding $g_{m}^{b}(F(x))=\left(\frac{1}{m+1}\left\{1-(F(x))^{m+1}\right\}\right)^{b}$ binomially in (8), we get

$$
\Phi_{j}(a, b)=\frac{1}{(m+1)^{b}} \sum_{u=0}^{b}(-1)^{u}\left(\begin{array}{l}
b \\
u
\end{array}\right) \int_{0}^{\infty} x^{j}[F(x)]^{a+u(m+1)} f(x) d x .
$$

Making the substitution $t=[F(x)]^{1 / \theta}$ in (9), we find that

$$
\Phi_{j}(a, b)=\frac{\theta}{(m+1)^{b}} \sum_{u=0}^{b}(-1)^{u}\left(\begin{array}{l}
b \\
u
\end{array}\right) \int_{0}^{1}[-\ln (1-t)]^{j / \alpha} t^{\theta[a+u(m+1)+1]-1} d t .
$$

On using the logarithmic expansion

$$
[-\ln (1-t)]^{j}=\left(\sum_{p=1}^{\infty} \frac{t^{p}}{p}\right)^{j}=\sum_{p=0}^{\infty} z_{p}(j) t^{j+p}, \quad|t|<1,
$$

where $z_{p}(j)$ is the coefficient of $t^{j+p}$ in the expansion of $\left(\sum_{p=1}^{\infty} \frac{t^{p}}{p}\right)^{j}$ (see Balakrishnan and Cohen (1991), p-44)), we get

$$
\begin{gathered}
\Phi_{j}(a, b)=\frac{\theta}{(m+1)^{b}} \sum_{p=0}^{\infty} \sum_{u=0}^{b}(-1)^{u}\left(\begin{array}{l}
b \\
u
\end{array}\right) z_{p}(j / \alpha) \int_{0}^{1} t^{\theta[a+u(m+1)+1]+(j / \alpha)+p-1} d t \\
=\frac{1}{(m+1)^{b}} \sum_{p=0}^{\infty} \sum_{u=0}^{b}(-1)^{u}\left(\begin{array}{l}
b \\
u
\end{array}\right) \frac{z_{p}(j / \alpha)}{[a+u(m+1)+1+((j / \alpha)+p) / \theta]} .
\end{gathered}
$$

Case II: when $m=-1$, we have

$$
\Phi_{j}(a, b)=\frac{0}{0} \text {, as } \sum_{u=0}^{b}(-1)^{u}\left(\begin{array}{l}
b \\
u
\end{array}\right)=0 \text {. }
$$

Since (12) is of the form $\frac{0}{0}$ at $m \neq 1$, therefore, we have

$$
\Phi_{j}(a, b)=A \sum_{u=0}^{b}(-1)^{u}\left(\begin{array}{l}
b \\
u
\end{array}\right) \frac{[a+u(m+1)+1+((j / \alpha)+p) / \theta]^{-1}}{(m+1)^{b}}
$$

where

$$
A=\sum_{p=0}^{\infty} z_{p}(j / \alpha)
$$

Differentiating numerator and denominator of (13) $b$ times with respect to $m$, we get

$$
=A \sum_{u=0}^{b}(-1)^{u+b}\left(\begin{array}{l}
b \\
u
\end{array}\right) \frac{u^{b}}{[a+u(m+1)+1+((j / \alpha)+p) / \theta]^{b+1}}, \quad b>0 .
$$

On applying L' Hospital rule, we have

$$
\lim _{m \rightarrow-1} \Phi_{j}(a, b)=A \sum_{u=0}^{b}(-1)^{u+b}\left(\begin{array}{l}
b \\
u
\end{array}\right) \frac{u^{b}}{[a+1+((j / \alpha)+p) / \theta]^{b+1}} .
$$

But for all integers $n \geq 0$ and for all real numbers $x$, we have Ruiz (1996) 


$$
\sum_{i=0}^{n}(-1)^{i}\left(\begin{array}{l}
n \\
i
\end{array}\right)(x-i)^{n}=n ! .
$$

Therefore,

$$
\sum_{u=0}^{b}(-1)^{u+b}\left(\begin{array}{l}
b \\
u
\end{array}\right) u^{b}=b !
$$

On substituting (16) in (14), we find that

$$
\Phi_{j}(a, b)=b ! \sum_{p=0}^{\infty} \frac{z_{p}(j / \alpha)}{[a+1+((j / \alpha)+p) / \theta]^{b+1}}, \quad m=-1 .
$$

Now substituting for $\Phi_{j}\left(\gamma_{r}-1, r-1\right)$ from (12) in (7) and simplifying, we obtain when $m \neq-1$

$$
\begin{aligned}
E\left[X^{* j-\alpha}(r, n, m, k)\right]=\frac{C_{r-1}}{(r-1) !(m+1)^{r-1}} \sum_{p=0}^{\infty} \sum_{u=0}^{r-1}(-1)^{u}\left(\begin{array}{c}
r-1 \\
u
\end{array}\right) & \\
& \times \frac{z_{p}((j / \alpha)-1)}{\left[\gamma_{r-u}+((j / \alpha)+p-1) / \theta\right]}
\end{aligned}
$$

and when $m=-1$, in view of (17) and (7), we have

$$
E\left[X^{* j-\alpha}(r, n,-1, k)\right]=E\left[\left(Z_{r}^{(k)}\right)^{j-\alpha}\right]=k^{r} \sum_{p=0}^{\infty} \frac{z_{p}((j / \alpha)-1)}{[k+((j / \alpha)+p-1) / \theta]^{r}},
$$

where $Z_{r}^{(k)}$ denote the $k$-th lower record value.

We obtain the recurrence relations for single moments of exponentiated Weibull distribution.

Theorem 1. For the distribution as given in (2) for $2 \leq r \leq n, n \geq 2$ and $k=1,2, \ldots$

$$
\begin{aligned}
E\left[X^{* j-\alpha}(r, n, m, k)\right] & =E\left[X^{* j-\alpha}(r-1, n, m, k)\right] \\
& +\frac{j-\alpha}{\alpha \theta \gamma_{r}}\left\{E\left[X^{* j-2 \alpha}(r, n, m, k)\right]-E\left[\phi\left(X^{*}(r, n, m, k)\right)\right]\right\},
\end{aligned}
$$

where

$$
\phi(x)=x^{j-2 \alpha} e^{x^{\alpha}}
$$

Proof. In view of Khan et al.(2008), note that

$$
\begin{aligned}
E\left[X^{* j-\alpha}(r, n, m, k)\right]-E\left[X^{* j-\alpha}(r-1, n, m, k)\right] & \\
& =-\frac{(j-\alpha) C_{r-1}}{\gamma_{r}(r-1) !} \int_{0}^{\infty} x^{j-\alpha-1}[F(x)]^{\gamma_{r}} g_{m}^{r-1}(F(x)) d x .
\end{aligned}
$$

By using (6) in (22), we get

$$
\begin{aligned}
E\left[X^{* j-\alpha}(r, n, m, k)\right]-E\left[X^{* j-\alpha}(r-1, n,\right. & m, k)] \\
& =\frac{j-\alpha}{\alpha \theta \gamma_{r}}\left\{\frac{C_{r-1}}{(r-1) !} \int_{0}^{\infty} x^{j-2 \alpha}[F(x)]^{\gamma_{r}-1} f(x) g_{m}^{r-1}(F(x)) d x\right. \\
& \left.-\frac{C_{r-1}}{(r-1) !} \int_{0}^{\infty} x^{j-2 \alpha} e^{x^{\alpha}}[F(x)]^{\gamma_{r}-1} f(x) g_{m}^{r-1}(F(x)) d x\right\}
\end{aligned}
$$


and hence the result given in (21).

Remark 1. Putting $m=0, k=1$, in (21), we obtain a recurrence relation for single moments of order statistics of the exponentiated Weibull distribution of the form

$$
E\left(X_{n-r+1: n}^{j-\alpha}\right)=E\left(X_{n-r+2: n}^{j-\alpha}\right)+\frac{j-\alpha}{\alpha \theta(n-r+1)}\left\{E\left(X_{n-r+1: n}^{j-2 \alpha}\right)-E\left(\phi\left(X_{n-r+1: n}\right)\right)\right\} .
$$

Replacing $(n-r+1)$ by $(r-1)$, we have

$$
E\left(X_{r: n}^{j-\alpha}\right)=E\left(X_{r-1: n}^{j-\alpha}\right)+\frac{j-\alpha}{\alpha \theta(r-1)}\left\{E\left(X_{r: n}^{j-2 \alpha}\right)-E\left(\phi\left(X_{r: n}\right)\right)\right\} .
$$

Remark 2. Setting $m=-1$ and $k \geq 1$ in (21), we get a recurrence relation for single moments of lower $k$ record values from exponentiated Weibull distribution in the form of

$$
E\left[\left(Z_{r}^{(k)}\right)^{j-\alpha}\right]=E\left[\left(Z_{r-1}^{(k)}\right)^{j-\alpha}\right]+\frac{j-\alpha}{\alpha \theta k}\left\{E\left[\left(Z_{r}^{(k)}\right)^{j-2 \alpha}\right]-E\left[\phi\left(Z_{r}^{(k)}\right)\right]\right\} .
$$

\section{Relation for Ratio Moments}

The explicit expressions for the product moments of $d g o s X^{* i-\alpha}(r, n, m, k)$ and $X^{* j-\alpha}(s, n, m, k), 1 \leq r<s \leq n$,

$$
\begin{aligned}
E\left[X^{* i-\alpha}(r, n, m, k) X^{* j-\alpha}(s, n, m, k)\right] & \\
& =\frac{C_{s-1}}{(r-1) !(s-r-1) !} \int_{0}^{\infty} \int_{0}^{x} x^{i-\alpha} y^{j-\alpha}[F(x)]^{m} f(x) g_{m}^{r-1}(F(x)) \\
& \times\left[h_{m}(F(y))-h_{m}(F(x))\right]^{s-r-1}[F(y)]^{\gamma_{s}-1} f(y) d y d x .
\end{aligned}
$$

On expanding $g_{m}^{r-1}(F(x))=\left(\frac{1}{m+1}\left\{1-(F(x))^{m+1}\right\}\right)^{r-1}$ binomially in (23), we get

$$
\begin{gathered}
E\left[X^{* i-\alpha}(r, n, m, k) X^{* j-\alpha}(s, n, m, k)\right]=\frac{C_{s-1}}{(r-1) !(s-r-1) !(m+1)^{r-1}} \\
\times \sum_{u=0}^{r-1}(-1)^{u}\left(\begin{array}{c}
r-1 \\
u
\end{array}\right) \int_{0}^{\infty} \int_{0}^{x} x^{i-\alpha} y^{j-\alpha}[F(x)]^{m+u(m+1)} f(x)\left[h_{m}(F(y))-h_{m}(F(x))\right]^{s-r-1}[F(y)]^{\gamma_{s}-1} f(y) d y d x \\
=\frac{C_{s-1}}{(r-1) !(s-r-1) !(m+1)^{r-1}} \sum_{u=0}^{r-1}(-1)^{u}\left(\begin{array}{c}
r-1 \\
u
\end{array}\right) \Phi_{i-\alpha, j-\alpha}\left(m+u(m+1), s-r-1, \gamma_{s}-1\right),
\end{gathered}
$$

where

$$
\Phi_{i, j}(a, b, c)=\int_{0}^{\infty} \int_{0}^{x} x^{i} y^{j}[F(x)]^{a} f(x)\left[h_{m}(F(y))-h_{m}(F(x))\right]^{b}[F(y)]^{c} f(y) d y d x .
$$

Case I: when $m \neq-1$

$$
\begin{aligned}
& \text { Expanding } \quad\left[h_{m}(F(y))-h_{m}(F(x))\right]^{b} \quad \text { binomially } \quad \text { in } \quad \text { (25) } \\
& h_{m}(F(y))-h_{m}(F(x))=g_{m}(F(y))-g_{m}(F(x)) \text {, we get } \\
& \Phi_{i, j}(a, b, c)=\frac{1}{(m+1)^{b}} \sum_{v=0}^{b}(-1)^{v}\left(\begin{array}{l}
b \\
v
\end{array}\right) \int_{0}^{\infty} x^{i}[F(x)]^{a+(b-v)(m+1)} f(x) I(x) d x
\end{aligned}
$$

where

$$
I(x)=\int_{0}^{x} y^{j}[F(y)]^{c+v(m+1)} f(y) d y .
$$

By setting $t=[F(y)]^{1 / \theta}$ in (27) and simplifying on the line of (12), we find that 


$$
I(x)=\sum_{p=0}^{\infty} \frac{z_{p}(j / \alpha)[F(x)]^{c+v(m+1)+1+((j / \alpha)+p) / \theta}}{[c+v(m+1)+1+\{(j / \alpha)+p\} / \theta]} .
$$

By substituting the expression of $I(x)$ in (26), we have

$$
\begin{aligned}
\Phi_{i, j}(a, b, c)=\frac{1}{(m+1)^{b}} \sum_{p=0}^{\infty} \sum_{v=0}^{b}(-1)^{v}\left(\begin{array}{l}
b \\
v
\end{array}\right) & \frac{z_{p}(j / \alpha)}{[c+v(m+1)+1+\{(j / \alpha)+p\} / \theta]} \\
& \times \int_{0}^{\infty} x^{i}[F(x)]^{a+c+b(m+1)+1+\{(j / \alpha)+p\} / \theta} f(x) d x .
\end{aligned}
$$

Again by setting $w=[F(x)]^{1 / \theta}$ in (28) and simplifying the resulting expression, we obtain

$$
\begin{aligned}
\Phi_{i, j}(a, b, c)=\frac{1}{(m+1)^{b}} \sum_{p=0}^{\infty} \sum_{q=0}^{\infty} \sum_{v=0}^{b}(-1)^{v}\left(\begin{array}{l}
b \\
v
\end{array}\right) \frac{z_{p}(j / \alpha)}{[c+v(m+1)+1+\{(j / \alpha)+p\} / \theta]} \\
\times \frac{z_{q}(i / \alpha)}{[a+c+b(m+1)+2+\{(i / \alpha)+(j / \alpha)+p+q\} / \theta]}
\end{aligned}
$$

Case II: when $m=-1$

$$
\Phi_{i, j}(a, b, c)=\frac{0}{0}, \text { as } \sum_{v=0}^{b}(-1)^{v}\left(\begin{array}{l}
b \\
v
\end{array}\right)=0 .
$$

Therefore, on applying L' Hospital rule and using (16), we find that

$$
\begin{aligned}
\lim _{m \rightarrow-1} \Phi_{i, j}(a, b, c)=b ! \sum_{p=0}^{\infty} \sum_{q=0}^{\infty} & \frac{z_{p}(j / \alpha)}{[c+1+\{(j / \alpha)+p\} / \theta]^{b+1}} \\
& \times \frac{z_{q}(i / \alpha)}{[a+c+2+\{(i / \alpha)+(j / \alpha)+p+q\} / \theta]} .
\end{aligned}
$$

Now on substituting for $\Phi_{i, j}\left(m+u(m+1), s-r-1, \gamma_{s}-1\right)$ from (29) in (24) and simplifying, we obtain when $m \neq-1$

$$
\begin{aligned}
E\left[X^{* i-\alpha}(r, n, m, k) X^{* j-\alpha}(s, n, m, k)\right]=\frac{C_{s-1}}{(r-1) !(s-r-1) !(m+1)^{s-2}} \\
\quad \times \sum_{p=0}^{\infty} \sum_{q=0}^{\infty} \sum_{u=0}^{r-1} \sum_{v=0}^{s-r-1}(-1)^{u+v}\left(\begin{array}{c}
r-1 \\
u
\end{array}\right)\left(\begin{array}{c}
s-r-1 \\
v
\end{array}\right) \frac{z_{p}((j / \alpha)-1)}{\left[\gamma_{s-v}+\{(j / \alpha)+p-1\} / \theta\right]} \\
\quad \times \frac{z_{q}((i / \alpha)-1)}{\left[\gamma_{r-u}+\{(i / \alpha)+(j / \alpha)+p+q-2\} / \theta\right]}
\end{aligned}
$$

and when $m=-1$, in view of (30) and (25), we have

$$
\begin{aligned}
& E\left[X^{* i-\alpha}(r, n,-1, k) X^{* j-\alpha}(s, n,-1, k)\right]=E\left[\left(Z_{r}^{(k)}\right)^{i-\alpha}\left(Z_{s}^{(k)}\right)^{j-\alpha}\right] \\
& =(\theta k)^{s} \sum_{p=0}^{\infty} \sum_{q=0}^{\infty} \frac{z_{p}((j / \alpha)-1) z_{q}((i / \alpha)-1)}{[\theta k+(j / \alpha)+p-1]^{s-r}[\theta k+(i / \alpha)+(j / \alpha)+p+q-2]^{r}} .
\end{aligned}
$$

Remark 3. At $j=\alpha$ in (31), we have 


$$
E\left[X^{* i-\alpha}(r, n, m, k)\right]=\frac{C_{r-1}}{(r-1) !(m+1)^{r-1}} \sum_{p=0}^{\infty} \sum_{u=0}^{r-1}(-1)^{u}\left(\begin{array}{c}
r-1 \\
u
\end{array}\right) \frac{z_{p}((i / \alpha)-1)}{\left[\gamma_{r-u}+\{(i / \alpha)+p-1\} / \theta\right]}
$$

which is the exact expression for single moment as given in (18).

Theorem 2. For the distribution as given in (2), for $1 \leq r<s \leq n, n \geq 2$ and $k=1,2, \ldots$

$$
\begin{aligned}
E\left[X^{* i-\alpha}(r, n, m, k) X^{* j-\alpha}(s, n, m, k)\right]-E\left[X^{* i-\alpha}(r, n, m, k) X^{* j-\alpha}(s-1, n, m, k)\right] & \\
& =\frac{j-\alpha}{\alpha \theta \gamma_{s}}\left\{E\left[X^{* i-\alpha}(r, n, m, k) X^{* j-2 \alpha}(s, n, m, k)\right]-E\left[\phi\left(X^{*}(r, n, m, k) X^{*}(s, n, m, k)\right)\right]\right\},
\end{aligned}
$$

where

$$
\phi(x, y)=x^{i-\alpha} y^{j-2 \alpha} e^{y^{\alpha}} .
$$

Proof. In view of Khan et al.(2008), note that

$$
\begin{aligned}
& E\left[X^{* i-\alpha}(r, n, m, k) X^{* j-\alpha}(s, n, m, k)\right]- E\left[X^{* i-\alpha}(r, n, m, k) X^{* j-\alpha}(s-1, n, m, k)\right] \\
&=-\frac{(j-\alpha) C_{s-1}}{\gamma_{s}(r-1) !(s-r-1) !} \int_{0}^{\infty} \int_{0}^{x} x^{i-\alpha} y^{j-\alpha-1}[F(x)]^{m} f(x) g_{m}^{r-1}(F(x)) \\
& \times\left[h_{m}(F(y))-h_{m}(F(x))\right]^{s-r-1}[F(y)]^{\gamma_{s}} d y d x .
\end{aligned}
$$

On using relation (6) in (35), we get

$$
\begin{gathered}
E\left[X^{* i-\alpha}(r, n, m, k) X^{* j-\alpha}(s, n, m, k)\right]-E\left[X^{* i-\alpha}(r, n, m, k) X^{* j-\alpha}(s-1, n, m, k)\right] \\
=-\frac{(j-\alpha) C_{s-1}}{\alpha \theta \gamma_{s}(r-1) !(s-r-1) !}\left\{\int_{0}^{\infty} \int_{0}^{x} x^{i-\alpha} y^{j-2 \alpha} e^{y^{\alpha}}[F(x)]^{m} f(x) g_{m}^{r-1}(F(x))\right. \\
\times\left[h_{m}(F(y))-h_{m}(F(x))\right]^{s-r-1}[F(y)]^{\gamma_{s}-1} f(y) d y d x \\
\left.-\int_{0}^{\infty} \int_{0}^{x} x^{i-\alpha} y^{j-2 \alpha}[F(x)]^{m} f(x) g_{m}^{r-1}(F(x))\left[h_{m}(F(y))-h_{m}(F(x))\right]^{s-r-1}[F(y)]^{\gamma_{s}-1} f(y) d y d x\right\}
\end{gathered}
$$

and hence the result given in (34).

Remark 4. Putting $m=0, k=1$ in (34), we obtain recurrence relations for product moments of order statistics of the exponentiated Weibull distribution in the form

$$
\begin{aligned}
E\left(X_{n-r+1: n}^{i-\alpha} X_{n-s+1: n}^{j-\alpha}\right)-E\left(X_{n-r+1: n}^{i-\alpha} X_{n-s+2: n}^{j-\alpha}\right) & =\frac{j-\alpha}{\alpha \theta(n-s+1)} \\
& \times\left\{E\left(X_{n-r+1: n}^{i-\alpha} X_{n-s+1: n}^{j-2 \alpha}\right)-E\left(\phi\left(X_{n-r+1: n} X_{n-s+1: n}\right)\right)\right\} .
\end{aligned}
$$

That is

$$
E\left(X_{r: n}^{i-\alpha} X_{s: n}^{j-\alpha}\right)-E\left(X_{r-1: n}^{i-\alpha} X_{s: n}^{j-\alpha}\right)=\frac{i-\alpha}{\alpha \theta(r-1)}\left\{E\left(X_{r: n}^{i-\alpha} X_{s: n}^{j-2 \alpha}\right)-E\left(\phi\left(X_{r: n} X_{s: n}\right)\right)\right\} .
$$

Remark 5. Setting $m=-1$ and $k \geq 1$, in (34), we obtain the recurrence relations for product moments of lower $k$ record values from exponentiated Weibull distribution in the form 


$$
\begin{aligned}
& E\left[\left(Z_{r}^{(k)}\right)^{i-\alpha}\left(Z_{s}^{(k)}\right)^{j-\alpha}\right]-E\left[\left(Z_{r}^{(k)}\right)^{i-\alpha}\left(Z_{s-1}^{(k)}\right)^{j-\alpha}\right] \\
& \quad=\frac{j-\alpha}{\alpha \theta k}\left\{E\left[\left(Z_{r}^{(k)}\right)^{i-\alpha}\left(Z_{s}^{(k)}\right)^{j-2 \alpha}\right]-E\left[\phi\left(Z_{r}^{(k)}\right)\left(Z_{s}^{(k)}\right)\right]\right\} .
\end{aligned}
$$

Remark 6. At $i=0$, Theorem 2 reduces to Theorem 1 .

\section{Special Cases of Ratio and Inverse Moments}

i) Putting $m=0, k=1$ in (18), the explicit formula for single moments of order statistics of the exponentiated Weibull distribution can be obtained as

$$
E\left(X_{n-r+1: n}^{j-\alpha}\right)=C_{r: n} \sum_{p=0}^{\infty} \sum_{u=0}^{r-1}(-1)^{u}\left(\begin{array}{c}
r-1 \\
u
\end{array}\right) \frac{z_{p}((j / \alpha)-1)}{[n-r+1+u+\{(j / \alpha)+p-1\} / \theta]^{r}} .
$$

That is

$$
E\left(X_{r: n}^{j-\alpha}\right)=C_{r: n} \sum_{p=0}^{\infty} \sum_{u=0}^{n-r}(-1)^{u}\left(\begin{array}{c}
n-r \\
u
\end{array}\right) \frac{z_{p}((j / \alpha)-1)}{[r-1+u+((j / \alpha)+p-1) / \theta]^{r}},
$$

where

$$
C_{r: n}=\frac{n !}{(r-1) !(n-r) !} \text {. }
$$

ii) Putting $k=1$ in (19), we deduce the explicit expression for the moments of lower record values from the exponentiated Weibull distribution as

$$
E\left(X_{L(r)}^{j}\right)=\sum_{p=0}^{\infty} \frac{z_{p}((j / \alpha)-1)}{[1+\{(j / \alpha)+p-1\} / \alpha]^{r}} .
$$

iii) Putting $m=0, k=1$ in (31), the explicit formula for the product moments of order statistics of the exponentiated Weibull distribution is obtained as

$$
\begin{aligned}
E\left(X_{n-r+1: n}^{i-\alpha} X_{n-s+1: n}^{j-\alpha}\right) & =C_{r, s: n} \sum_{p=0}^{\infty} \sum_{q=0}^{\infty} \sum_{u=0}^{r-1} \sum_{v=0}^{s-r-1}(-1)^{u+v}\left(\begin{array}{c}
r-1 \\
u
\end{array}\right)\left(\begin{array}{c}
s-r-1 \\
v
\end{array}\right) \\
& \times \frac{z_{p}((j / \alpha)-1) z_{q}((i / \alpha)-1)}{[n-s+1+v+\{(j / \alpha)+p-1\} / \theta][n-r+1+u+\{(i / \alpha)+(j / \alpha)+p+q-2\} / \theta]} .
\end{aligned}
$$

That is

$$
\begin{aligned}
E\left(X_{r: n}^{i-\alpha} X_{s: n}^{j-\alpha}\right)=C_{r, s: n} \sum_{p=0}^{\infty} \sum_{q=0}^{\infty} \sum_{u=0}^{n-s} \sum_{v=0}^{s-r-1}(-1)^{u+v}\left(\begin{array}{c}
n-s \\
u
\end{array}\right)\left(\begin{array}{c}
s-r-1 \\
v
\end{array}\right) & \\
& \times \frac{z_{p}((i / \alpha)-1) z_{q}((j / \alpha)-1)}{[r-1+v+\{(j / \alpha)+p-1\} / \alpha][s-1+u+\{(i / \alpha)+(j / \alpha)+p+q-2\} / \theta]},
\end{aligned}
$$

where

$$
C_{r, s: n}=\frac{n !}{(r-1) !(s-r-1) !(n-s) !} .
$$

iv) Putting $k=1$ in (32), the explicit formula for the product moments of lower record values for the exponentiated Weibull distribution can be obtained as 


$$
E\left[X_{L(r)}^{i-\alpha} X_{L(s)}^{j-\alpha}\right]=\theta^{s} \sum_{p=0}^{\infty} \sum_{q=0}^{\infty} \frac{z_{p}((j / \alpha)-1) z_{q}((i / \alpha)-1)}{[\theta+(j / \alpha)+p-1]^{s-r}[\theta+(i / \alpha)+(j / \alpha)+p+q-2]^{r}}
$$

\section{Characterization}

Let $X^{*}(r, n, m, k), r=1,2, \ldots, n$ be dgos from a continuous population with $d f \quad F(x)$ and $p d f f(x)$, then the conditional $p d f$ of $X^{*}(s, n, m, k)$ given $X^{*}(r, n, m, k)=x, 1 \leq r<s \leq n$, in view of (4) and (5), is

$$
\begin{aligned}
& f_{X^{*}(s, n, m, k) \mid X^{*}(r, n, m, k)}(y \mid x)=\frac{C_{s-1}}{(s-r-1) ! C_{r-1}}[F(x)]^{m-\gamma_{r}+1} \\
& \times\left[\left(h_{m}(F(y))-h_{m}(F(x))\right]^{s-r-1}[F(y)]^{\gamma_{s}-1} f(y), \quad y<x, \quad m \neq-1\right. \\
& f_{Z_{s}^{(k)} \mid Z_{r}^{(k)}}(y \mid x)=\frac{k^{s-r}}{(s-r-1) !}[\ln F(x)-\ln F(y)]^{s-r-1} \\
& \times\left(\frac{F(y)}{F(x)}\right)^{k-1} \frac{f(y)}{F(x)} d y, \quad y<x, \quad m=-1
\end{aligned}
$$

Theorem 3. Let $X$ be a non-negative random variable having an absolutely continuous $d f \quad F(x)$ with $F(0)=0$ and $0<F(x)<1$ for all $x>0$, then

$$
\begin{gathered}
E\left[\xi\left\{X^{*}(s, n, m, k)\right\} \mid X^{*}(l, n, m, k)=x\right]=\sum_{p=1}^{\infty} \frac{\left(1-e^{-x^{\alpha}}\right)^{p}}{p} \prod_{j=1}^{s-l}\left(\frac{\gamma_{r+j}}{\gamma_{r+j}+(p / \theta)}\right), \\
l=r, \quad r+1, \quad m \neq-1 .
\end{gathered}
$$

$$
\begin{aligned}
E\left[\xi\left(Z_{s}^{(k)}\right) \mid Z_{l}^{(k)}=x\right]=\sum_{p=1}^{\infty}\left(\frac{k}{k+(p / \theta)}\right)^{s-l}\left(1-e^{-x^{\alpha}}\right)^{p} / p, & \\
& l=r, r+1, \quad m=-1 .
\end{aligned}
$$

where

$$
\xi(y)=y^{\alpha} .
$$

if and only if

$$
F(x)=\left(1-e^{-x^{\alpha}}\right)^{\theta}, \quad x>0, \quad \alpha, \theta>0 .
$$

Proof. When $m \neq-1$, we have from (36) for $s>r+1$

$$
\begin{aligned}
& E[\xi\{X(s, n, m, k)\} \mid X(r, n, m, k)=x]=\frac{C_{s-1}}{(s-r-1) ! C_{r-1}(m+1)^{s-r-1}} \\
& \times \int_{0}^{x} y^{\alpha}\left[1-\left(\frac{F(y)}{F(x)}\right)^{m+1}\right]^{s-r-1}\left(\frac{F(y)}{F(x)}\right)^{\gamma_{s}-1} \frac{f(y)}{F(x)} d y .
\end{aligned}
$$

By setting $u=\frac{F(y)}{F(x)}=\left(\frac{1-e^{-y^{\alpha}}}{1-e^{-x^{\alpha}}}\right)^{\theta}$ from (2) in (40), we obtain 


$$
\begin{aligned}
E[\xi\{X(s, n, m, k)\} \mid X(r, n, m, k) & =x]=\frac{C_{s-1}}{(s-r-1) ! C_{r-1}(m+1)^{s-r-1}} \\
& \times \int_{0}^{1}\left[-\ln \left\{1-\left(1-e^{-x^{\alpha}}\right) u^{1 / \theta}\right\}\right] u^{\gamma_{s}-1}\left(1-u^{m+1}\right)^{s-r-1} d u \\
& =\frac{C_{s-1}}{(s-r-1) ! C_{r-1}(m+1)^{s-r-1}} \sum_{p=1}^{\infty} \frac{\left(1-e^{-x^{\alpha}}\right)^{p}}{p} \int_{0}^{1} u^{(p / \theta)+\gamma_{s}-1}\left(1-u^{m+1}\right)^{s-r-1} d u .
\end{aligned}
$$

Again by setting $t=u^{m+1}$ in (41), we get

$$
\begin{aligned}
E[\xi\{X(s, n, m, k)\} \mid X(r, n, m, k)=x] & =\frac{C_{s-1}}{(s-r-1) ! C_{r-1}(m+1)^{s-r}} \\
& \times \sum_{p=1}^{\infty} \frac{\left(1-e^{-x^{\alpha}}\right)^{p}}{p} \int_{0}^{1} t^{\frac{p+\theta k}{\theta(m+1)}+n-s-1}(1-t)^{s-r-1} d t \\
= & \frac{C_{s-1}}{C_{r-1}(m+1)^{s-r}} \sum_{p=1}^{\infty} \frac{\left(1-e^{-x^{\alpha}}\right)^{p}}{p} \frac{\Gamma\left(\frac{p+\theta k}{\theta(m+1)}+n-s\right)}{\Gamma\left(\frac{p+\theta k}{\theta(m+1)}+n-r\right)} \\
= & \frac{C_{s-1}}{C_{r-1}} \sum_{p=1}^{\infty} \frac{\left(1-e^{-x^{\alpha}}\right)^{p}}{p \prod_{j=1}^{s-r}\left(\gamma_{r+j}+(p / \theta)\right)},
\end{aligned}
$$

where

$$
\frac{C_{s-1}}{C_{r-1}}=\prod_{j=1}^{s-r} \gamma_{r+j}
$$

and hence the result given in (38).

To prove sufficient part, we have from (36) and (38)

$$
\begin{aligned}
\frac{C_{s-1}}{(s-r-1) ! C_{r-1}(m+1)^{s-r-1}} \int_{0}^{x} y\left[(F(x))^{m+1}-(F(y))^{m+1}\right]^{s-r-1} \\
\times[F(y)]^{\gamma_{s}-1} f(y) d y=[F(x)]^{\gamma_{r+1}} H_{r}(x),
\end{aligned}
$$

where

$$
H_{r}(x)=\sum_{p=1}^{\infty} \frac{\left(1-e^{-x^{\alpha}}\right)^{p}}{p} \prod_{j=1}^{s-r}\left(\frac{\gamma_{r+j}}{\gamma_{r+j}+(p / \theta)}\right) .
$$

Differentiating (42) both sides with respect to $x$, we get

$$
\begin{aligned}
\frac{C_{s-1}[F(x)]^{m} f(x)}{(s-r-2) ! C_{r-1}(m+1)^{s-r-2}} \int_{0}^{x} y\left[(F(x))^{m+1}-(F(y))^{m+1}\right]^{s-r-2}[F(y)]^{\gamma_{s}-1} f(y) d y \\
=H_{r}^{\prime}(x)[F(x)]^{\gamma_{r+1}}+\gamma_{r+1} H_{r}(x)[F(x)]^{\gamma_{r+1}-1} f(x)
\end{aligned}
$$

or 


$$
\begin{array}{r}
\gamma_{r+1} H_{r+1}(x)[F(x)]^{\gamma_{r+2}+m} f(x) \\
=H_{r}^{\prime}(x)[F(x)]^{\gamma_{r+1}}+\gamma_{r+1} H_{r}(x)[F(x)]^{\gamma_{r+1}-1} f(x) .
\end{array}
$$

Therefore,

$$
\frac{f(x)}{F(x)}=\frac{H_{r}^{\prime}(x)}{\gamma_{r+1}\left[H_{r+1}(x)-H_{r}(x)\right]}=\frac{\alpha \theta x^{\alpha-1} e^{-x^{\alpha}}}{1-e^{-x^{\alpha}}},
$$

where

$$
\begin{aligned}
& H_{r}^{\prime}(x)=\alpha x^{\alpha-1} e^{-x^{\alpha}} \sum_{p=1}^{\infty}\left(1-e^{-x^{\alpha}}\right)^{p-1} \prod_{j=1}^{s-r}\left(\frac{\gamma_{r+j}}{\gamma_{r+j}+(p / \theta)}\right), \\
& H_{r+1}(x)-H_{r}(x)=\frac{1}{\theta \gamma_{r+1}} \sum_{p=1}^{\infty}\left(1-e^{-x^{\alpha}}\right)^{p} \prod_{j=1}^{s-r}\left(\frac{\gamma_{r+j}}{\gamma_{r+j}+(p / \theta)}\right) .
\end{aligned}
$$

Integrating both the sides of (43) with respect to $x$ between $(0, y)$, the sufficiency part is proved.

For the case when $m=-1$, from (37) on using the transformation $u=\frac{F(y)}{F(x)}=\left(\frac{1-e^{-y^{\alpha}}}{1-e^{-x^{\alpha}}}\right)^{\theta}$, we find that

$$
E\left[\xi\left(Z_{s}^{(k)}\right) \mid Z_{l}^{(k)}=x\right]=A^{*} \int_{0}^{1}(-\ln u)^{s-r-1} u^{k+(p / \theta)-1} d u,
$$

where

$$
A^{*}=\frac{k^{s-r}}{(s-r-1) !} \sum_{p=1}^{\infty}\left(1-e^{-x^{\alpha}}\right)^{p} / p
$$

We have Gradshteyn and Ryzhik (2007, p-551)

$$
\int_{0}^{1}(-\ln x)^{\mu-1} x^{v-1} d x=\frac{\Gamma \mu}{v^{\mu}}, \quad \mu>0, \quad v>0 .
$$

On using (45) in (44), we have the result given in (39).

Sufficiency part can be proved on the lines of case $m \neq-1$.

Theorem 4. Let $X$ be a non-negative random variable having an absolutely continuous $d f \quad F(x)$ with $F(0)=0$ and $0<F(x)<1$ for all $x>0$, then

$$
\begin{aligned}
E\left[X^{* j-\alpha}(r, n, m, k)\right]=E\left[X^{* j-\alpha}(r-1, n, m, k)\right] & -\frac{j-\alpha}{\alpha \theta \gamma_{r}} E\left[\phi\left(X^{*}(r, n, m, k)\right)\right] \\
& +\frac{j-\alpha}{\alpha \theta \gamma_{r}} E\left[X^{* j-2 \alpha}(r, n, m, k)\right]
\end{aligned}
$$

if and only if

$$
F(x)=\left(1-e^{-x^{\alpha}}\right)^{\theta}, \quad x>0, \quad \alpha>0, \theta>0 .
$$

Proof. The necessary part follows immediately from (21). On the other hand if the recurrence relation in (46) is satisfied. In view of Khan et al.(2008), note that 


$$
\begin{aligned}
& -\frac{(j-\alpha) C_{r-1}}{\gamma_{r}(r-1) !} \int_{0}^{\infty} x^{j-\alpha-1}[F(x)]^{\gamma_{r}} g_{m}^{r-1}(F(x)) d x= \\
& -\frac{(j-\alpha) C_{r-1}}{\alpha \theta \gamma_{r}(r-1) !} \int_{0}^{\infty} x^{j-2 \alpha} e^{x^{\alpha}}[F(x)]^{\gamma_{r}-1} f(x) g_{m}^{r-1}(F(x)) d x \\
& \quad+\frac{(j-\alpha) C_{r-1}}{\alpha \theta \gamma_{r}(r-1) !} \int_{0}^{\infty} x^{j-2 \alpha}[F(x)]^{\gamma_{r}-1} f(x) g_{m}^{r-1}(F(x)) d x \\
& \frac{(j-\alpha) C_{r-1}}{\gamma_{r}(r-1) !} \int_{0}^{\infty} x^{j-\alpha}[F(x)]^{\gamma_{r}-1} g_{m}^{r-1}(F(x)) d x\left\{\frac{F(x)}{x f(x)}-\frac{x^{-\alpha}}{\alpha \theta} e^{x^{\alpha}}+\frac{1}{\alpha \theta}\right\} d x=0
\end{aligned}
$$

Now applying a generalization of the Müntz-Szász Theorem (Hwang and Lin, 1984) to (48), we get

$$
\frac{F(x)}{x f(x)}=\frac{\left(e^{x^{\alpha}}-1\right)}{\alpha \theta x^{\alpha}}
$$

which proves that

$$
F(x)=\left(1-e^{-x^{\alpha}}\right)^{\theta}, \quad x>0, \quad \alpha, \theta>0 .
$$

Theorem 5. Suppose an absolutely continuous (with respect to Lebesgue measure) random variable $X$ has the $d f$ $F(x)$ and $p d f f(x)$ for $0<x<\infty$, such that $f^{\prime}(x)$ and $E(X \mid X \leq x)$ exist for all $x, 0<x<\infty$, then

$$
E(X \mid X \leq x)=g(x) \eta(x),
$$

where

$$
\eta(x)=\frac{f(x)}{F(x)}
$$

and

$$
g(x)=\frac{1-e^{-x^{\alpha}}}{\alpha \theta x^{\alpha-2} e^{-x^{\alpha}}}-\frac{\int_{0}^{x}\left(1-e^{-u^{\alpha}}\right)^{\theta} d u}{\alpha \theta x^{\alpha-1}\left(1-e^{-x^{\alpha}}\right)^{\theta-1} e^{-x^{\alpha}}},
$$

if and only if

$$
f(x)=\alpha \theta x^{\alpha-1}\left(1-e^{-x^{\alpha}}\right)^{\theta-1} e^{-x^{\alpha}}, \quad x>0, \alpha, \theta>0 .
$$

Proof. In view of Ahsanullah, et al. (2016) and (1), we have

$$
E(X \mid X \leq x)=\frac{\alpha \theta}{F(x)} \int_{0}^{x} u^{\alpha}\left(1-e^{-u^{\alpha}}\right)^{\theta-1} e^{-u^{\alpha}} d u .
$$

Integrating (50) by parts treating $\left(u^{\alpha-1}\left(1-e^{-u^{\alpha}}\right)^{\theta-1} e^{-u^{\alpha}}\right)$ for integration and rest of the integrant for differentiation, we get

$$
E(X \mid X \leq x)=\frac{1}{F(x)}\left\{x\left(1-e^{-x^{\alpha}}\right)^{\theta}-\int_{0}^{x}\left(1-e^{-u^{\alpha}}\right)^{\theta} d u\right\} .
$$

After multiplying and dividing by $f(x)$ in (51), we have the result given (49).

To prove sufficient part, we have from (49)

$$
\frac{1}{F(x)} \int_{0}^{x} u f(u) d u=\frac{g(x) f(x)}{F(x)}
$$


or $\quad \int_{0}^{x} u f(u) d u=g(x) f(x)$.

Differentiating (52) on both the sides with respect to $x$, we find that

$$
x f(x)=g^{\prime}(x) f(x)+g(x) f^{\prime}(x) .
$$

Therefore,

$$
\begin{gathered}
\frac{f^{\prime}(x)}{f(x)}=\frac{x-g^{\prime}(x)}{g(x)} \quad \text { [Ahsanullah, et al. (2016)] } \\
=\frac{\alpha(\theta-1) x^{\alpha-1} e^{-x^{\alpha}}}{1-e^{-x^{\alpha}}}+\frac{\alpha-1}{x}-x^{\alpha-1} e^{-x^{\alpha}}
\end{gathered}
$$

where

$$
g^{\prime}(x)=x+g(x)\left(\frac{\alpha(\theta-1) x^{\alpha-1} e^{-x^{\alpha}}}{1-e^{-x^{\alpha}}}+\frac{\alpha-1}{x}-x^{\alpha-1} e^{-x^{\alpha}}\right) .
$$

Integrating both the sides in (53) with respect to $x$, we get

$$
f(x)=c x^{\alpha-1}\left(1-e^{-x^{\alpha}}\right)^{\theta-1} e^{-x^{\alpha}} .
$$

It is known that

$$
\int_{0}^{\infty} f(x) d x=1
$$

Thus,

$$
\frac{1}{c}=\int_{0}^{\infty} x^{\alpha-1}\left(1-e^{-x^{\alpha}}\right)^{\theta-1} e^{-x^{\alpha}} d x=\frac{1}{\alpha \theta},
$$

which proved that

$$
f(x)=\alpha \theta x^{\alpha-1}\left(1-e^{-x^{\alpha}}\right)^{\theta-1} e^{-x^{\alpha}}, \quad x>0, \alpha, \theta>0 .
$$

\section{Conclusion}

This paper demonstrates the explicit expressions for ratio and inverse moment for single moment as well as product moment through lower (dual) generalized order statistics. Some recurrence relations for single and product moments of dgos from the exponentiated Weibull distribution have been established. We calculate for both cases $E[x / y]$ and $E[y / x]$ on $j<\alpha$ and $i<\alpha$ respectively. Further, conditional expectation of function, recurrence relation and truncated expectation of dgos has been utilized to obtain a characterization of the exponentiated Weibull distribution and some important results are deduced.

\section{Acknowledgments}

The authors acknowledge with thanks the concrete suggestions and comments of the learned Referees, which led to an overall improvement of the paper.

\section{References}

1. Ahsanullah, M. (2004): A characterization of the uniform distribution by dual generalized order statistics. Comm. Statist. Theory Methods, 33, 2921-2928.

2. Ahsanullah, M. (2005): On lower generalized order statistics and a characterization of power function distribution. Stat. Methods, 7, 16-28. 
3. Ahsanullah, M., Shakil, M., Golam Kibria, B.M. (2016): Characterization of continuous distribution by truncated moment. Journal of Modern Applied Statistical Method, 15, 316-331.

4. Burkschat, M., Cramer, E. and Kamps, U. (2003): Dual generalized order statistics. Metron, LXI, 13-26.

5. Balakrishnan, N. and Cohen, A.C. (1991): Order Statistics and Inference: Estimation Methods. Academic Press, San Diego.

6. Gradshteyn, I.S. and Ryzhik, I.M. (2007): Table of Integrals, Series, and Products. Academic Press, New York.

7. Hwang, J.S. and Lin, G.D. (1984): On a generalized moments problem II. Proc. Amer. Math. Soc., 91, 577-580.

8. Khan, R.U., Anwar, Z. and Athar, H. (2008): Recurrence relations for single and product moments of dual generalized order statistics from exponentiated Weibull distribution. Aligarh J. Statist., 28, 37-45.

9. Khan, A.H., Anwar, Z. and Chisti, S. (2010): Characterization of continuous distributions through conditional expectation of functions of dual generalized order statistics. Pakistan J. Statist., 26, 615-628.

10. Khan, A.H., Khan, R.U. and Yaqub, M. (2006): Characterization of continuous distributions through conditional expectation of function of generalized order statistics. J. Appl. Probab. Stat., 1, 115-131.

11. Khan, R.U. and Kumar, D. (2010): On moments of lower generalized order statistics from exponentiated Pareto distribution and its characterization. Appl. Math. Sci. (Ruse), 4, 2711-2722.

12. Khan, R.U. and Kumar, D. (2011): Expectation identities of lower generalized order statistics from generalized exponential distribution and a characterization. Math. Methods Statist., 20, 150-157.

13. Khan, R.U. and Khan, M.A. (2015): Dual generalized order statistics from family of $J$-shaped distribution and its characterization. Journal of King Saud University-Science, 27, 285-291.

14. Mbah, A.K. and Ahsanullah, M. (2007): Some characterization of the power function distribution based on lower generalized order statistics. Pakistan J. Statist., 23, 139-146.

15. Mudholkar, G.S. and Hutson, A.D. (1996): The exponentiated Weibull family: some properties and a flood data application. Comm. Statist. Theory Methods, 25, 3059-3083.

16. Mudholkar, G.S., Srivastava, D.K. and Freimer, M. (1995): The exponentiated Weibull family. Technometrics, 37, 436-445.

17. Nassar, M.M. and Eissa, F.H. (2003): On exponentiated Weibull distribution. Comm. Statist. Theory Methods, 32, 1317-1336.

18. Pawlas, P. and Szynal, D. (2001): Recurrence relations for single and product moments of lower generalized order statistics from the inverse Weibull distributions. Demonstratio Math., XXXIV, 353-358.

19. Ruiz, S.M. (1996): An algebraic identity leading to Wilson's theorem. The Mathematical Gazette, 80, 579-582.

20. Shawky, A.I. and Bakoban, R.A. (2008): Characterization from exponentiated gamma distribution based on record values. J. Statist. Theory Appl., 7, 263-277.

21. Mudholkar, G.S. and Srivastava, D.K. (1993): Exponentiated Weibull family for analyzing bathtub failure rate data. IEEE Transactions on Reliability, 42, 299-302.

22. Pal, M., Ali, M. M., and Woo, J. (2006): Exponentiated Weibull distribution. Statistica, 66(2), 139-147.

23. Jiang R. and Murthy DNP (1999): The exponentiated Weibull family: a graphical approach. IEEE Trans Reliab, $48,68-72$.

24. Nadarajah, S., Cordeiro, G. M. and Ortega, E. M. M. (2013): The exponentiated Weibull distribution: a survey, Statistical Papers, 54, 839-877. 